J. Nonlinear Sci. Vol. 11: p. 237 (2001)

DOI: $10.1007 / \mathrm{s} 00332-001-0438-8$

(C) 2001 Springer-Verlag New York Inc.

\title{
Erratum \\ A Theory of Exact Solutions for Annular Viscous Blobs
}

\author{
Darren Crowdy ${ }^{1}$ and Saleh Tanveer ${ }^{2}$ \\ 1 Department of Mathematics, Imperial College of Science, Technology and Medicine, 180 \\ Queen's Gate, London, United Kingdom \\ e-mail: d.crowdy@ic.ac.uk \\ 2 Department of Mathematics, Ohio State University, Columbus, OH 43210, USA \\ e-mail: tanveer@math.ohio-state.edu
}

Received August 3, 2001; accepted August 14, 2001

Communicated by $\mathrm{T}$. Fokas

The particular example given in $\$ 6$ of Crowdy and Tanveer [1] to illustrate the general theory is in error because it is not consistent with the original assumptions underlying the theory. The theory developed in earlier sections assumes that a single-valued analytic function $F(\zeta, t)$ can be found in the annulus $\rho(t)<|\zeta|<1$ when the integration constants $A_{O}(t)$ and $A_{I}(t)$ in equations (16) and (17) are set equal to each other. It has since been pointed out by Richardson [2], and we agree with him, that this assumption is self-consistent when the solutions represent interfaces that have a rotational symmetry, which the example in $\$ 6$ does not have. This particular example should be disregarded. Once initial conditions are chosen with such rotational symmetries, the general theory given in [1] is still applicable and useful and can reveal interesting features of the physical problem as shown in a recent paper by Crowdy [3], where examples based on the theory of [1] are calculated and investigated. The theory of [1] is different in both approach and formulation from the one subsequently developed by Richardson [2] for the same mathematical problem of surface tension driven Stokes flow of a doubly-connected region.

\section{References}

[1] D. G. Crowdy \& S. Tanveer, A theory of exact solutions for annular viscous blobs, J. Nonlin. Sci., 8, 375-400 (1998).

[2] S. Richardson, Plane Stokes flow with time-dependent free boundaries in which the fluid occupies a doubly-connected region, Eur. J. Appl. Math., 11, 249-269 (2000).

[3] D. G. Crowdy, Viscous sintering of unimodal and bimodal cylindrical packings with shrinking pores, submitted to Eur. J. Appl. Math. 